\title{
Multicriteria Analysis in Additive Manufacturing: An ELECTRE-MOr Based Approach
}

\author{
Paula DRUMOND ${ }^{\mathrm{a}}$, Marcio Pereira BASÍLIO ${ }^{\mathrm{b}}$, Igor Pinheiro de Araújo COSTA ${ }^{\mathrm{c}, 1}$, \\ Daniel Augusto de Moura PEREIRA ${ }^{\mathrm{d}}$, Carlos Francisco Simões GOMES ${ }^{\mathrm{c}}$ and Marcos \\ dos SANTOS ${ }^{\mathrm{a}}$ \\ ${ }^{a}$ Military Institute of Engineering, Brazil \\ ${ }^{b}$ Military Police of the State of Rio de Janeiro, Brazil \\ ${ }^{c}$ Federal Fluminense University, Brazil \\ ${ }^{d}$ Federal University of Campina Grande, Brazil
}

\begin{abstract}
D printing technologies define the essence of Additive Manufacturing and make possible the agile production of customized parts from different materials, with lower unit cost and waste generation. Currently, one of the most widespread $3 \mathrm{D}$ printer technologies is the Fused Deposition Modeling (FDM) type, which is the object of this paper. The choice of 3D printing equipment depends on the alignment of the purpose of use and technical knowledge to consider certain requirements. Therefore, this choice can be time-consuming and/or imprecise. In this sense, this work aimed to classify FDM-type 3D printer models by applying the ELECTREMOr method, a Multi-criteria Decision Aiding (MCDA) method. As a result, based on a categorization between classes, the FABER 10 alternative was the only one that presented class A performance in all evaluated scenarios, based on criteria defined by the experts consulted in this study.
\end{abstract}

Keywords. Multi-criteria Decision Aiding (MCDA), ELECTRE-MOr, Additive Manufacturing, Decision Making.

\section{Introduction}

Additive Manufacturing (AM) is the technology capable of transforming a complex geometry model, elaborated through a computational system, into a product without the need for a long production planning process. The manufacture of parts happens with the overlap of material layers, added one by one in a three-dimensional printing area, after software configurations [1]. According to [2], AM can be considered as the manufacturing paradigm of the future due to its ability to meet changes in capacity and functionality quickly and efficiently.

AM has gained wide diffusion in a number of applications based on the layer manufacturing technique in recent years [3]. However, it is a challenge to select an

${ }^{1}$ Corresponding Author, Igor Pinheiro de Araújo COSTA, Department of Production Engineering, Federal Fluminense University, Niterói, Brazil; E-mail: costa_igor@id.uff.br.

This research was financially supported by Military Police of the State of Rio de Janeiro. 
Appropriate AM process for a particular customer or company. This is because the result may vary due not only to different materials and printers, but also to different parameters and post-processes [4].

According to [5], selecting an appropriate process or machine to manufacture an end-use product is an important issue in AM design. One of the many types of approaches to process selection is based on Multi-criteria Decision Aiding (MCDA). In this context, MCDA consists of a set of formal approaches that explicitly seek to take into account multiple criteria to help stakeholders and groups explore important decisions [6]. These decisions generally involve various conflicting objectives, cloudy types of nonrepeatable uncertainties, accumulated costs and benefits for various individuals, companies, groups and other organizations [7].

Despite the diversity of multi-criteria approaches, methods and techniques, the basic components of MCDA are a finite or infinite set of actions (alternatives, solutions, courses of action, etc.), at least two criteria and at least one Decision Maker (DM). Given these basic elements, MCDA is an activity that assists in decision making, especially in terms of choice, classification or ordering of actions [8].

This paper aims proposes, through the application of the ELECTRE-MOr MCDA method, an algorithm capable of supporting the strategic process to classify 3D printing equipment for AM. The axiomatic model of the ELECTRE-MOr method allows the evaluation and obtaining of the weights of the criteria, through qualitative pairwise analysis by specialists in AM, and the distribution of alternatives into predefined classes. The method was chosen because it distributes alternatives in classes, which allows the DM to select one or more options that are allocated to the highest classes.

This research is divided into six sections. After this introduction, section 2 presents the literature review; section 3 structures the problem and the methodology, while section 4 deals with the main characteristics of the ELECTRE-MOr method; section 5 demonstrates its application and results; finally, section 6 concludes this research.

\section{Literature Review}

With Industry 4.0 trends covering physical and virtual media, AM represents a way for the realization of complex geometries designed on computers [9]. Widely known as 3D Printing, AM has been spreading around the world due to the possibility of developing customized products quickly and with minimal material waste, which positively impacts the production chain [1].

In this context, the Fused Deposition Modeling (FDM) printer uses heat to melt a thermoplastic filament and then generates 3D item from digital designs [10], presenting an economic process, mainly in the construction of items with complex geometry or almost any shape [11].

The academic literature presents some MCDA methods' applications in problems related to AM, such as: evaluation of the MA machines capacity, based on the ability to produce a standard component [12]; a proposed hybrid modeling to help users in the AM options selection process [4].

Khamhong et al. [13] used the Fuzzy Analytic Hierarchy Process (Fuzzy AHP) methodology to analyze criteria weights for factors related to the selection of $3 \mathrm{D}$ printers. Raigar et al. [14] proposed a hybrid multicriteria methodology to select an appropriate AM process from available processes. The Best Worst Method (BWM) was applied to 
determine the optimal weights of the criteria, and the Proximity Indexed Value (PIV) method was used to classify the available AM processes.

According to [15], the costs of the machine and the material are the significant parameters, which play an important role in estimating the cost of the AM. The authors applied the BWM method to select the appropriate material for an AM project, aiming to reduce the costs inherent to the project.

According to [16], although the options are plentiful, selecting an appropriate choice of AM is not a trivial task. The authors applied the Combinative Distance-Based Assessment (CODAS) method under the Pythagorean Fuzzy (PF) to assist in the selection of the 3D printing technology best suited to the needs of the supply chain in a given company.

The literature review, although not exhaustive, revealed several MCDA applications to support the decision-making process in AM-related problems. However, it was verified the lack of models for sorting alternatives in classes, as is the proposal of this paper.

The modeling presented in this research distributes the alternatives in predefined categories, presenting ordinal entry of weights, multiple DMs and evaluation of quantitative and qualitative criteria. Furthermore, the ELECTRE-MOR method presents two ways to obtain the lower bounds of predefined classes, resulting in wo alternative distributions. This feature provides greater transparency and robustness to the decisionmaking process.

\section{Methodology}

Considering a real decision-making problem, uncertainty is intrinsic. As suggested by [17], the methods used as decision-aid should enable an integrated algorithm, enabling the evaluation of qualitative and quantitative data. That is, tools should be able to structure and analyze variables in situations where it is not possible to define a precise numeric entry. In the decision analysis, the presence of uncertainty regarding the evaluation of a problem is recurrent, considering that the information obtained may be lacking in complete or certainty data [18].

During the analysis of the data referring to the case study of this paper, we found that, among the diverse available MCDA tools, the ELECTRE-MOr method has good adherence to the problem, because it distributes the alternatives in predefined classes and allows obtaining the criteria weights by ordinal entries, considering the opinion of multiple DMs.

Therefore, this work aims to propose an action plan for classification of FDM 3D printers, based on the application of the ELECTRE-MOr method, which facilitates the expression of preference relationships for DMs, contributing to transparent and reliable decision making.

According to the classification proposed by [19], this research can be characterized as mixed qualitative-quantitative, combining case study and mathematical modeling [20]. $\mathrm{AM}$ is the object of research, as previously presented in Sections 1 and 2. The background and details about the case study are presented in Sections 4 and 5, respectively. 


\section{The ELECTRE-MOr method}

The MCDA methods are very useful to support the decision-making process in several situations, because they consider value judgments and not only technical issues, to evaluate alternatives to solve real problems, presenting a highly multidisciplinarity [21]. These methods have been employed to support the decision-making process in several recent complex problems, as presented in [22-26].

The ELECTRE MOr, proposed by [27], is a multicriteria sorting method with ordinal weight input that includes multiple DMs and distributes the alternatives into predefined categories. The ELECTRE MOr procedures are developed in two stages. To obtain the weights and evaluate qualitative criteria, the ELECTRE-MOr method uses an adaptation of the SAPEVO method [28,29], transforming ordinal preferences of criteria into a vector of criteria weights and integrating the vector criteria of different DMs.

According to [27], ELECTRE-MOr has the following features:

- The elicitation of weights of the criteria by an ordinal form, since this is not an easy task for a DM, because it requires establishing a precise numerical value for such parameters as the importance coefficients of criteria;

- The distribution in classes is carried out through two procedures:

1. Optimistic: it consists of comparing the alternative successively to alternative $\mathrm{b}$, from the last profile (category, class);

2. Pessimistic: it consists of comparing the alternative a successively to the alternative $b$, starting from the first profile (category, class), which is the most demanding classification.

- Two ways of obtaining the lower limits of the classes (bh and bn), which provides 4 different sorts ( 2 optimistic and 2 pessimistic), allowing a more robust and reliable sensitivity analysis of the results.

\section{Case Study}

Given the numerous options of FDM 3D printers found in the market, we consulted three specialists (DMs) in the AM field, with extensive experience and knowledge in 3D printers, to designate the alternative components of this research. For possible alternatives, printers that have similarities between construction pattern (equipment and finished product) and price were considered, so that there was no inequality between the selected alternatives. Thus, it was concluded that eight printers would be evaluated: ENDER 3, ENDER 3 PRO, DAVINCI PRO 1.0, GI3, S2, PRIME ONE V3, GRABER I 3 and FABER 10.

Also, the DMs defined six relevant criteria that relate to the choice of equipment and that can influence the quality of the 3D printed parts (Table 1):

Table 1. Establishment of outranking relationships.

\begin{tabular}{lll}
\hline Acronym & Criteria & Monotonicity \\
\hline $\mathrm{C}_{1}$ & Print speed $(\mathrm{mm} / \mathrm{s})$ & Profit \\
$\mathrm{C}_{2}$ & Printing area $\left(\mathrm{mm}^{2}\right)$ & Profit \\
$\mathrm{C}_{3}$ & Price $(\mathrm{R} \$)$ & Cost \\
$\mathrm{C}_{4}$ & Layer resolution $(\mathrm{mm})$ & Cost \\
$\mathrm{C}_{5}$ & Warranty (Months) & Profit \\
$\mathrm{C}_{6}$ & Energy consumption $(\mathrm{w})$ & Cost \\
\hline
\end{tabular}


The monotonicity of the criteria was determined, that is, whether they are cost or benefit criteria, in order to minimize or maximize them, respectively, when incorporated into the ELECTRE-MOr method. In order to obtain the weights of the criteria (through pairwise comparisons between them), interviews were conducted through video conference with the three specialists together to establish the degrees by consensus.

\subsection{Evaluation of alternatives}

Table 2 shows the performance matrix, with the alternatives being evaluated in the light of each criterion. To obtain data referring to criteria $\mathrm{C}_{1}, \mathrm{C}_{2}, \mathrm{C}_{4}, \mathrm{C}_{5}$ and $\mathrm{C}_{6}$, we considered parameters contained in the equipment's technical manuals. To obtain the price $\left(\mathrm{C}_{3}\right)$, a market research was carried out.

Table 2. Performance matrix.

\begin{tabular}{lllllll}
\hline Alternatives / Criteria & $\mathbf{C}_{\mathbf{1}}$ & $\mathbf{C}_{\mathbf{2}}$ & $\mathbf{C}_{\mathbf{3}}$ & $\mathbf{C}_{\mathbf{4}}$ & $\mathbf{C}_{\mathbf{5}}$ & $\mathbf{C}_{\mathbf{6}}$ \\
\hline ENDER 3 & 100 & 48.400 & -2.199 & -0.050 & 6 & -340 \\
ENDER 3 PRO & 100 & 48.40 & -2.599 & -0.050 & 6 & -340 \\
DAVINCI PRO 1.0 & 120 & 40.000 & -10.000 & -0.075 & 12 & -200 \\
GI3 & 200 & 40.000 & -3.100 & -0.050 & 12 & -360 \\
S2 & 150 & 40.000 & -5.550 & -0.050 & 12 & -350 \\
PRIME ONE V3 & 75 & 40.000 & -2.350 & -0.080 & 3 & -360 \\
GRABER I3 & 80 & 40.000 & -1.950 & -0.050 & 12 & -360 \\
FABER 10 & 200 & 90.000 & -3.685 & -0.070 & 12 & -270 \\
bh $_{3}$ & 169 & 77.500 & -3.963 & -0.058 & 10 & -240 \\
bh $_{2}$ & 138 & 65.000 & -5.975 & -0.065 & 8 & -280 \\
bh $_{1}$ & 106 & 52.500 & -7.988 & -0.073 & 5 & -320 \\
bn $_{3}$ & 150 & 48.400 & -2.199 & -0.070 & 12 & -270 \\
bn $_{2}$ & 100 & 40.000 & -2.599 & -0.075 & 6 & -350 \\
bn $_{1}$ & 80 & 40.000 & -3.685 & -0.080 & 3 & -360 \\
q & 15 & 2.000 & 100 & 0.025 & 6 & 15 \\
p & 20 & 4.000 & 500 & 0.050 & 12 & 50 \\
v & 25 & 10.000 & 10.000 & 0.075 & 10 & 200 \\
Weights of criteria & 0.92 & 1.21 & 2.00 & 1.29 & 0.72 & 0.45 \\
\hline
\end{tabular}

It is observed that the criterion with the highest weight after analysis of the three specialists was the price, followed by the resolution of the layer and printing area. The energy consumption criterion was considered as the least important by analysts.

The monotonic cost criteria were represented with negative values in the performance matrix. The Weak Preference (q), Strict Preference (p) and Veto (v) thresholds were defined by consensus among the experts. In addition, it was established that the eight alternatives would be distributed into four classes.

\subsection{Results obtained}

After applying all the steps of ELECTRE-MOr, optimistic and pessimistic classifications were obtained for the two forms of distribution ( $b h$ and bn). In consensus among the authors and specialists, a cutting level $\lambda$ of 0.6 was established, because it presented a good discrimination in the classification of alternatives. Thus, the distribution of alternatives in the four predefined classes is obtained (Table 3).

Table 3. Results obtained after application of the ELECTRE-MOr method.

\begin{tabular}{lllll}
\hline $\boldsymbol{\lambda}=\mathbf{0 , 6}$ & \multicolumn{2}{c}{ bh } & & bn \\
Alternatives & Pessimist & Optimistic & Pessimist & Optimistic \\
\hline ENDER 2 & $\mathrm{C}$ & $\mathrm{C}$ & $\mathrm{B}$ & $\mathrm{B}$ \\
ENDER 3 PRO & $\mathrm{C}$ & $\mathrm{C}$ & $\mathrm{B}$ & $\mathrm{B}$ \\
\hline
\end{tabular}




\begin{tabular}{lllll}
\hline DAVINCI PRO 1.0 & D & D & B & B \\
GI3 & D & A & A & A \\
S2 & D & C & A & A \\
PRIME ONE V3 & D & D & C & C \\
GRABER I3 & D & D & B & B \\
FABER 10 & A & A & A & A \\
\hline
\end{tabular}

In view of the classifications obtained through the method, it was observed that the FABER 10 alternative was unique with the classification corresponding to Class $A$ in both scenarios (pessimistic and optimistic) within the two distributions (bh and bn). Therefore, this is the most suitable 3D printer to be purchased in the face of the conditions addressed.

In the analysis of bh, it is noted that only FABER 10 achieved the best classification in both scenarios. Analyzing the bn procedure, it is perceived that the alternatives GI3, $\mathrm{S} 2$ also reached the highest class, but GI3 obtained a bad result in the pessimistic scenario of bh and S2 had poor performance in both bh scenarios.

Among the reasons that led FABER 10 to be the best alternative, it is possible to observe the good overall performance in the criteria, highlighting the large printing area (third criterion of higher weight) of $90,000 \mathrm{~mm}^{2}$ and the printing speed of $200 \mathrm{~mm} / \mathrm{s}$, which justifies the good performance of the alternative in the proposed analysis.

\section{Conclusions}

This article aimed to support the classification process of 3D printers of the FDM type, in order to evaluate the alternatives selected in the light of established criteria to reach a result based on a multicriteria model. The application of the ELECTRE-MOr method considered the evaluation of three different specialists, based on the criteria in question and the weights derived from their evaluations, having interfered on the final result.

We highlight that the method proved to be efficient for the proposed analysis, allowing the entry of ordinal weights, considering the opinion of multiple DMs, both in obtaining the weights, as well as in elicitation of the preference and veto thresholds. Besides, the presentation of two pessimistic and optimistic ordinations allowed us to verify the behavior of alternatives in various scenarios, with the possibility of choosing several parameters of analysis, either by considering the highest number of occurrences of classifications, as was done in this paper, or by considering only one of the two forms of distribution. As a suggestion for future work, it is proposed that other relevant and/or alternative criteria be included, modifying the cutting level, and that 3D printers of other types be analyzed.

\section{References}

[1]. GIBSON I., ROSEN DW., STUCKER B. Additive Manufacturing Technologies: 3D Printing, rapid prototyping and direct digital manufacturing. New York: Springer; 2015.

[2]. Sonar HC, Khanzode V V, Akarte MM. Ranking of Additive Manufacturing Implementation Factors using Analytic Hierarchy Process (AHP). J Inst Eng Ser C [Internet]. 2021;102(2):421-6.

[3]. Ransikarbum K, Khamhong P. Integrated Fuzzy Analytic Hierarchy Process and Technique for Order of Preference by Similarity to Ideal Solution for Additive Manufacturing Printer Selection. J Mater Eng Perform [Internet]. 2021.

[4]. Wang Y, Zhong RY, Xu X. A decision support system for additive manufacturing process selection using a hybrid multiple criteria decision-making method. Rapid Prototyp J [Internet]. 2018;24(9):1544-53.

[5]. Qin Y, Qi Q, Scott PJ, Jiang X. An additive manufacturing process selection approach based on fuzzy 
Archimedean weighted power Bonferroni aggregation operators. Robot Comput Integr Manuf . 2020;64.

[6]. Belton V, Stewart T. Multiple criteria decision analysis: an integrated approach. Springer Science \& Business Media; 2002.

[7]. Keeney RL, Raiffa H, Meyer RF. Decisions with multiple objectives: preferences and value trade-offs. Cambridge university press; 1993.

[8]. Greco S, Figueira J, Ehrgott M. Multiple Criteria Decision Analysis: State of art surveys. Vol. 37. Springer; 2016.

[9]. Oh Y, Witherell P, Lu Y, Sprock T. Nesting and scheduling problems for additive manufacturing: A taxonomy and review. Addit Manuf [Internet]. 2020;36.

[10].Joo Y, Shin I, Ham G, Abuzar SM, Hyun S-M, Hwang S-J. The advent of a novel manufacturing technology in pharmaceutics: Superiority of fused deposition modeling 3D printer. J Pharm Investig. 2020;50(2):131-45.

[11].Tan DK, Maniruzzaman M, Nokhodchi A. Advanced pharmaceutical applications of hot-melt extrusion coupled with fused deposition modelling (FDM) 3D printing for personalised drug delivery. Pharmaceutics. 2018;10(4):203.

[12].Roberson DA, Espalin D, Wicker RB. 3D printer selection: A decision-making evaluation and ranking model. Virtual Phys Prototyp [Internet]. 2013;8(3):201-12.

[13].Khamhong P, Yingviwatanapong C, Ransikarbum K. Fuzzy Analytic Hierarchy Process (AHP)-based Criteria Analysis for 3D Printer Selection in Additive Manufacturing. In: RI2C 2019 - 2019 Research, Invention, and Innovation Congress [Internet]. 2019.

[14].Raigar J, Sharma VS, Srivastava S, Chand R, Singh J. A decision support system for the selection of an additive manufacturing process using a new hybrid MCDM technique. Sadhana - Acad Proc Eng Sci [Internet]. 2020;45(1).

[15].Palanisamy M, Pugalendhi A, Ranganathan R. Selection of suitable additive manufacturing machine and materials through best-worst method (BWM). Int J Adv Manuf Technol. 2020;107(5-6):2345-62.

[16].Büyüközkan G, Göçer F. Assessment of additive manufacturing technology by pythagorean fuzzy CODAS [Internet]. Vol. 1029, Advances in Intelligent Systems and Computing. 2020. p. 959-68.

[17].Malloy TF, Zaunbrecher VM, Batteate CM, Blake A, Carroll WF, Corbett CJ, et al. Advancing alternative analysis: Integration of decision science. Environ Health Perspect. 2017;125(6):1-12.

[18].Dong Y, Liu Y, Liang H, Chiclana F, Herrera-Viedma E. Strategic weight manipulation in multiple attribute decision making. Omega (United Kingdom). 2018;75:1339-51.

[19].Creswell JW, Creswell JD. Research design: Qualitative, quantitative, and mixed methods approaches. Sage publications; 2017.

[20].Bertrand JWM, Fransoo JC. Operations management research methodologies using quantitative modeling. Int J Oper Prod Manag. 2002;22(2):241-64.

[21].Santos M dos, Quintal RS, Paixão AC da, Gomes CFS. Simulation of Operation of an Integrated Information for Emergency Pre-Hospital Care in Rio de Janeiro Municipality. Procedia Comput Sci [Internet]. 2015;55:931-8.

[22].Costa IP de A, Maêda SM do N, Teixeira LFH de S de B, Gomes CFS, Santos M dos. Choosing a hospital assistance ship to fight the Covid-19 pandemic. Rev Saude Publica [Internet]. 2020;54.

[23].Tenório FM, dos Santos M, Gomes CFS, Araujo J de C. Navy Warship Selection and Multicriteria Analysis: The THOR Method Supporting Decision Making. In: International Joint conference on Industrial Engineering and Operations Management [Internet]. Springer; 2020. p. 27-39.

[24].Moreira MÂL, Costa IP de A, Pereira MT, dos Santos M, Gomes CFS, Muradas FM. PROMETHEESAPEVO-M1 a Hybrid Approach Based on Ordinal and Cardinal Inputs: Multi-Criteria Evaluation of Helicopters to Support Brazilian Navy Operations. Algorithms [Internet]. 2021 Apr 27;14(5):140.

[25].Oliveira AS, Gomes CFS, Clarkson CT, Sanseverino AM, Barcelos MRS, Costa IPA, et al. Multiple Criteria Decision Making and Prospective Scenarios Model for Selection of Companies to Be Incubated. Algorithms [Internet]. 2021 Mar 30;14(111).

[26].Santos M dos; , Costa IP de A, Gomes CFS. Multicriteria decision-making in the selection of warships: a new approach to the AHP method. Int J Anal Hierarchy Process [Internet]. 2021 May 19;13(1).

[27].Costa IP de A, Sanseverino AM, Barcelos MR dos S, Belderrain MCN, Gomes CFS, Santos M dos. Choosing flying hospitals in the fight against the COVID-19 pandemic: structuring and modeling a complex problem using the VFT and ELECTRE-MOr methods. IEEE Lat Am Trans [Internet]. 2021 Jun;19(6):1099-106.

[28].Gomes CFS, Santos M dos, Teixeira LFH de S de B, Sanseverino AM, Barcelos MR dos S. SAPEVO-M: a group multicriteria ordinal ranking method. Pesqui Operacional [Internet]. 2020;40.

[29].Gomes L, Mury A-R, Gomes CFS. Multicriteria ranking with ordinal data. Syst Anal. 1997;27(2):13946. 